\title{
Characterization of multiqubit pure-state entanglement
}

\author{
Zeqian Chen* \\ State Key Laboratory of Magnetic Resonance and Atomic and \\ Molecular Physics and United Laboratory of Mathematical Physics, \\ Wuhan Institute of Physics and Mathematics, Chinese Academy of Sciences, \\ 30 West District, Xiao-Hong-Shan, P.O.Box 71010, Wuhan 430071, China \\ Quanhua Xu丹 \\ Laboratoire de Mathématique, Université de Franche-Comté, \\ 16, Route de Gray, 25030 Besançon Cedex, France
}

(Dated: November 10, 2018)

\begin{abstract}
A necessary and sufficient entanglement criterion based on variances of Mermin-Klyshko's Bell operators is proved for multiqubit pure states. Contrary to Bell's inequalities, entangled pure states strictly satisfy a quadratic inequality but product ones can attain the equality under some local unitary transformations, which can be obtained by solving a quadratic maximum problem. This presents a characterization of multiqubit pure-state entanglement.
\end{abstract}

PACS numbers: 03.67.Mn, 03.65.Ud

Entanglement plays a crucial role in quantum communication, cryptograph, and computation [1]. The characterization of entanglement is one of the fundamental problems in quantum information theory [2]. Although there are some characterizations of entanglement for bipartite systems [3, 4] and many entanglement witnesses for testing multipartite entanglement [5], among which the most well known one is the violation of Bell's inequalities [6] that was originally designed to rule out various kinds of local hidden variable theories based on Einstein, Podolsky, and Rosen's (EPR's) notion of local realism [7, 8], simple necessary and sufficient conditions are not known for multipartite entanglement, even in the simplest case of multiqubits [9]. In particular, Żukowski et al [10] show that there are some generalized GHZ states of $n>2$ qubits that do not violate any Bell inequality for two dichotomic observables per qubit, including the inequality involving the mean values of Mermin-Klyshko's (MK) Bell operators. Since any pure entangled state of two qubits violates a Clauser-HorneShimony-Holt (CHSH) 11] inequality (Gisin's theorem [3]), that is, the CHSH inequality characterizes the purestate entanglement of two qubits, Scarani and Gisin 12 wrote that "this analysis suggests that MK inequalities, and more generally the family of Bell's inequalities with two observables per qubit, may not be the 'natural' generalizations of the CHSH inequality to more than two qubits".

However, in this article we find a necessary and sufficient entanglement criterion based on variances of MK's Bell operators for multiqubit pure states. This can be regarded as a generalization of Gisin's theorem from two qubits to $n$ qubits, although it cannot be straightfor-

*Electronic address: zqchen@wipm.ac.cn

${ }^{\dagger}$ Electronic address: quanhua.xu@math.univ-fcomte.fr wardly generalized to all multiqubit systems for the case of the Bell inequalities of two dichotomic observables per site involving the mean values of observables. Our criterion presents an operational characterization of multiqubit pure-state entanglement, because it is based on inequalities for variances of observables. Contrary to the Bell inequalities, entangled pure states strictly satisfy the inequality but product ones can attain the equality under some local unitary transformations, which can be obtained by solving a quadratic maximum problem. Our result sheds considerable new light on relationships between multipartite entanglement and Bell's operators.

Let us give a brief review of MK Bell operators and the associated Mermin-Klyshko inequalities. The MK Bell operators of $n$ qubits is defined recursively $(n \geq 2)$. Let $\vec{a}_{j} \vec{\sigma}_{j}, \vec{a}_{j}^{\prime} \vec{\sigma}_{j}$ denote spin observables on the $j$-th qubit, $j=1, \ldots, n$, where all $\vec{a}_{j}, \vec{a}_{j}^{\prime}$ are unit vectors in $\mathbb{R}^{3}$ and $\vec{\sigma}_{j}=\left(\sigma_{x}^{j}, \sigma_{y}^{j}, \sigma_{z}^{j}\right)$ is the Pauli matrices on the $j$-th qubit. Denote by $\mathcal{B}_{1}=\vec{a}_{1} \vec{\sigma}_{1}$ and $\mathcal{B}_{1}^{\prime}=\vec{a}_{1}^{\prime} \vec{\sigma}_{1}$. Define

$\mathcal{B}_{n}=\mathcal{B}_{n-1} \otimes \frac{1}{2}\left(\vec{a}_{n} \vec{\sigma}_{n}+\vec{a}_{n}^{\prime} \vec{\sigma}_{n}\right)+\mathcal{B}_{n-1}^{\prime} \otimes \frac{1}{2}\left(\vec{a}_{n} \vec{\sigma}_{n}-\vec{a}_{n}^{\prime} \vec{\sigma}_{n}\right)$,

where $\mathcal{B}_{n}^{\prime}$ denotes the same expression $\mathcal{B}_{n}$ but with all the $\vec{a}_{j}$ and $\vec{a}_{j}^{\prime}$ exchanged. $\mathcal{B}_{n}$ is called the MK Bell operator of $n$ qubits. Assuming "local realism" [7, 8], one concludes the Mermin-Klyshko inequality of $n$ qubits as follows,

$$
\left\langle\mathcal{B}_{n}\right\rangle \leq 1
$$

which can be violated by quantum mechanics [6]

By convention, we adopt the notation $\left|0^{n}\right\rangle=|0 \cdots 0\rangle$ and $\left|1^{n}\right\rangle=|1 \cdots 1\rangle$. For a pure state $|\psi\rangle$ of $n$ qubits, the variance of $\mathcal{B}_{n}$ on $|\psi\rangle$ is defined by

$$
\Delta\left(|\psi\rangle, \mathcal{B}_{n}\right)=\left\langle\psi\left|\mathcal{B}_{n}^{2}\right| \psi\right\rangle-\left\langle\psi\left|\mathcal{B}_{n}\right| \psi\right\rangle^{2} .
$$

Recall that when all the $\vec{a}_{j}$ and $\vec{a}_{j}^{\prime}$ are in the $x-$ $y$ plane and $\vec{a}_{j}$ 's are distributed with angles $(j-$ 
1) $(-1)^{n+1} 2 \pi /(2 n)$ with respect to the $x$-axis and $\vec{a}_{j} \perp \vec{a}_{j}^{\prime}$, the associated MK Bell operator has the following spectral decomposition 12 ]

$$
\hat{\mathcal{B}}_{n}=2^{(n-1) / 2}\left(\left|\mathrm{GHZ}_{+}\right\rangle\left\langle\mathrm{GHZ}_{+}|-| \mathrm{GHZ}_{-}\right\rangle\left\langle\mathrm{GHZ}_{-}\right|\right),
$$

where $\left|\mathrm{GHZ}_{ \pm}\right\rangle=\frac{1}{\sqrt{2}}\left(\left|0^{n}\right\rangle \pm\left|1^{n}\right\rangle\right)$ are GHZ's states [13]. Then, it is easy to check that $\Delta\left(\left|0^{n}\right\rangle, \hat{\mathcal{B}}_{n}\right)=2^{n-1}$. Moreover, for every product state $|\psi\rangle=\left|\psi_{1}\right\rangle \cdots\left|\psi_{n}\right\rangle$ of $n$ qubits, there is a local unitary transformation $U=U_{1} \otimes \cdots \otimes U_{n}$ such that $U|\psi\rangle=\left|0^{n}\right\rangle$. Hence,

$$
\Delta\left(|\psi\rangle, U^{\dagger} \hat{\mathcal{B}}_{n} U\right)=2^{n-1}
$$

where $U^{\dagger}$ denotes the adjoint operator of $U$. This leads the following

Theorem.- A pure state $|\psi\rangle$ of $n$ qubits is entangled if and only if

$$
\Delta\left(|\psi\rangle, U^{\dagger} \hat{\mathcal{B}}_{n} U\right)<2^{n-1},
$$

for every local unitary transformations $U$ such that $\left\langle 0^{n}|U| \psi\right\rangle$ and $\left\langle 1^{n}|U| \psi\right\rangle$ are both real numbers.

Furthermore, $|\psi\rangle$ is entangled whenever there is one $U$ satisfying Eq.(6) and solving the maximum problem

$$
\max \left(\left\langle 0^{n}|U| \psi\right\rangle^{2}+\left\langle 1^{n}|U| \psi\right\rangle^{2}\right)
$$

where the maximum is taken over all local unitary transformations so that $\left\langle 0^{n}|U| \psi\right\rangle$ and $\left\langle 1^{n}|U| \psi\right\rangle$ are both nonnegative numbers.

Proof.-The sufficiency of Theorem is shown above. To prove the necessity, suppose that there were $\mathcal{B}_{n}=U^{\dagger} \hat{\mathcal{B}}_{n} U$ for some $U$ so that $\Delta\left(|\psi\rangle, \mathcal{B}_{n}\right)=2^{n-1}$ and both $\left\langle 0^{n}|U| \psi\right\rangle$ and $\left\langle 1^{n}|U| \psi\right\rangle$ are real numbers. Since $\left\|\mathcal{B}_{n}\right\| \leq 2^{(n-1) / 2}$, it is concluded that

$$
\begin{gathered}
\mathcal{B}_{n}^{2}|\psi\rangle=2^{n-1}|\psi\rangle, \\
\left\langle\psi\left|\mathcal{B}_{n}\right| \psi\right\rangle=0 .
\end{gathered}
$$

As proved in [14], Eq.(8) implies that

$$
U|\psi\rangle=\alpha\left|0^{n}\right\rangle+\beta\left|1^{n}\right\rangle .
$$

Since $\alpha=\left\langle 0^{n}|U| \psi\right\rangle$ and $\beta=\left\langle 1^{n}|U| \psi\right\rangle$ are both real numbers, it is concluded from Eqs. (4) and (9) that either $\alpha$ or $\beta$ is zero and hence $|\psi\rangle$ is unentangled.

Furthermore, if $|\psi\rangle$ is a product state, then the maximum value of Eq.(7) is one. Suppose that $\tilde{U}$ is a solution of Eq.(7), then $\tilde{U}|\psi\rangle$ is a product state of the form $\alpha\left|0^{n}\right\rangle+\beta\left|1^{n}\right\rangle$ with $\alpha, \beta$ being two nonnegative numbers and hence must be equal to either $\left|0^{n}\right\rangle$ or $\left|1^{n}\right\rangle$, from which one concludes that $\Delta\left(|\psi\rangle, \tilde{U}^{\dagger} \hat{\mathcal{B}}_{n} \tilde{U}\right)=2^{n-1}$. This completes the proof.

By our criterion, it is easy to test the entanglement of the generalized GHZ states

$$
|\Psi(\varphi)\rangle=\cos \varphi\left|0^{n}\right\rangle+\sin \varphi\left|1^{n}\right\rangle
$$

with $0 \leq \varphi \leq \pi / 4$. Indeed, since the identity $I=I_{2} \otimes$ $\cdots \otimes I_{2}$ is a solution of the maximum problem Eq.(7), where $I_{2}$ is the identity on one qubit, we obtain that

$$
\Delta\left(|\Psi(\varphi)\rangle, \hat{\mathcal{B}}_{n}\right)=2^{n-1} \cos ^{2} 2 \varphi
$$

This exactly detects the entanglement of $|\Psi(\varphi)\rangle$, which, however, cannot be tested by any Bell inequality of two dichotomic observables per site for sufficiently small parameters $\varphi$ when $n>2$, as shown in [10].

Evidently, our criterion reduces to a quadratic program consisting of maximizing a quadratic objective Eq.(7) under two linear inequalities $\left\langle 0^{n}|U| \psi\right\rangle,\left\langle 1^{n}|U| \psi\right\rangle \geq 0$ for all local unitary transformations of $n$ qubits. The complexity of solving this quadratic program will be studied in the future. A similar argument using convex optimization programs is presented in [15].

Although our criterion is more powerful than the Bell inequalities of two dichotomic observables per site for detecting the pure-state entanglement of $n$ qubits, it cannot be used to test quantum mechanics versus EPR's local realism from which the original Bell inequality arose [16]. Indeed, as shown in [17], the physical origins of EPR's local realism and quantum entanglement are different. For a multipartite system which, having interacted in past, are now spatially separated, EPR's local realism means that elements of physical reality for one subsystem should be independent on what are done with the others. In contrary, quantum entanglement refers only to quantum multipartite states, whether or not the individual subsystems are spatially separated. The violation of Bell's inequalities assuming EPR's local realism by suitable entangled states is therefore an interesting but indirect consequence of quantum entanglement.

In summary, we have shown a Gisin-type theorem for $n$ qubits in terms of variances of Mermin-Klyshko's Bell operators, which cannot be straightforwardly generalized to all $n$-qubit systems for the case of the Bell inequalities of two dichotomic observables per site involving the mean values of observables [10]. Entanglement criteria based on inequalities for variances of observables have been studied, mainly designed for continuous variable systems [18]. Recently, it has been shown that variances of observables can be detect entanglement also in finitedimensional systems [19]. Our result furthermore shed considerable light on quantum entanglement in terms of variances of the Bell operators of a special type. We expect that, similarly to the Bell inequalities based on mean values of the Bell-type operators, the variances of Belltype operators and its various ramifications will play an important role in quantum entanglement.

This work was supported by the National Natural Science Foundation of China under Grant No.10571176, the National Basic Research Programme of China under Grant No.2001CB309309, and also funds from Chinese Academy of Sciences. 
[1] C.H.Bennett, et al, Phys.Rev.Lett.70, 1895(1993); N.Gisin, G.Ribordy, W.Tittle, and H.Zbinden, Rev. Mod. Phys.74, 145(2002); M.Nielsen and I.Chuang, Quantum Computation and Quantum Information (Cambridge University Press, Cambridge, England, 2000).

[2] D.Bruß, J.Math.Phys.43(9), 4237(2002).

[3] N.Gisin, Phys.Lett. A 154, 201(1991).

[4] A.Peres, Phys.Rev.Lett.77, 1413(1996); M.Horodecki, P.Horodecki, and R.Horodecki, Phys.Lett.223, 1(1996); M.Horodecki and P.Horodecki, Phys.Rev.A 59, 4206(1999).

[5] A search for "multipartite entanglement" in tittle on www.arxiv.org/quant-ph yields 72 results.

[6] N.D.Mermin, Phys.Rev.Lett. 65, 1838(1990); A.V.Belinskii, D.N.Klyshko, Phys. Usp. 36, 653(1993); N.Gisin and H.Bechmann-Pasquinucci, Phys. Lett. A 246, 1(1998); for a review, see R.F.Werner and M.M.Wolf, Quantum Inf. Comp.1, 1(2001).

[7] A.Einstein, B.Podolsky, and N.Rosen, Phys. Rev.47, $777(1935)$.

[8] J.S.Bell, Physics (Long Island City, N.Y.)1, 195(1964).

[9] A Bell-type inequality for probabilities is presented in [J.L.Chen, C.Wu, L.C.Kwek, and C.H.Oh, Phys.Rev.Lett. 93, 140407(2004)], which can be violated by all pure entangled states of three qubits as shown by numerical results but without a rigorous proof.

[10] M.Żukowski, Č.Brukner, W.Laskowski, and M.Wieśniak, Phys.Rev.Lett.88, 210402(2002).

[11] J.F.Clauser, M.A.Horne, A.Shimony, R.A.Holt,
Phys.Rev.Lett. 23, 880(1969).

[12] V.Scarani and N.Gisin, J.Phys.A: Math.Gen.34, 6043(2001).

[13] D.M.Greenberger, M.A.Horne, and A.Zeilinger, in Bell's Theorem, Quantum Theory, and Conceptions of the Universe, edited by M.Kafatos (Kluwer, Dordrecht, 1989), p.69; N.D.Mermin, Am. J. Phys. 58, 731(1990).

[14] Z.Chen, Phys.Rev.Lett.93, 110403(2004).

[15] F.G.S.L.Brandão and R.O.Vianna, Phys.Rev.Lett.93, 220503(2004); J.Eisert, P.Hyllus, O.Gühne, and M.Curty, Phys.Rev.A 70, 062317(2004); A.C.Doherty, P.A.Parrilo, and F.M.Spedalieri, Phys.Rev.A 71, 032333(2005).

[16] According to EPR's local realism, MK Bell operators take only values \pm 1 . Then, their variances are less than or equal to one and display that there is no contradiction betweem quantum mechanics and EPR's local realism.

[17] S.M.Roy, Phys.Rev.Lett.94, 010402(2005).

[18] L.-M.Duan, et al., Phys.Rev.Lett.84, 2722(2000); R.Simon, Phys.Rev.Lett.84, 2726(2000); S.Mancini, et al., ibid. 88, 120401(2002); N.Korolkova, et al., Phys.Rev.A 65, 052306(2002); V.Giovannetti, et al., ibid. 67, 022320(2003); P.van Loock and A.Furusawa, ibid, 67, 052315(2003); G.Tóth, C.Simon, and J.I.Cirac, ibid. 68, 062310(2003).

[19] H.F.Hofmann and S.Takeuchi, Phys.Rev.A 68, 032103(2003); H.F.Hofmann, ibid. 68, 034307(2003); O.Gühne, Phys.Rev.Lett.92, 117903(2004). 